\title{
Minimum Weight Design of Sinusoidal Corrugated Web Beam Using Real-Coded Genetic Algorithms
}

\author{
Sudeok Shon, ${ }^{1}$ Sangwook Jin, ${ }^{1}$ and Seungjae Lee ${ }^{2}$ \\ ${ }^{1}$ Department of Architectural Engineering, Korea University of Technology and Education, Cheonan 31253, Republic of Korea \\ ${ }^{2}$ Interdisciplinary Program in Creative Engineering, Korea University of Technology and Education, Cheonan 31253, Republic of Korea \\ Correspondence should be addressed to Seungjae Lee; leeseung@koreatech.ac.kr
}

Received 16 November 2016; Accepted 26 December 2016; Published 6 March 2017

Academic Editor: Roman Lewandowski

Copyright (c) 2017 Sudeok Shon et al. This is an open access article distributed under the Creative Commons Attribution License, which permits unrestricted use, distribution, and reproduction in any medium, provided the original work is properly cited.

\begin{abstract}
Fundamental advantage of using corrugated web girder rather than plate girder reinforced with stiffeners is securing stability against shear buckling of web and unnecessary stiffeners despite the thinner web. Nonetheless, because shear buckling behavior of corrugated web is very complex, the design mechanism for beams and local, global, and interactive buckling problems should be considered in designing of its structural optimization for better economics and reasonableness. Therefore, this paper proposes a mathematical model for minimum weight design of sinusoidal web girder for securing better stability with smooth corrugation and aims at developing its optimum design program. The constraints for the optimum design were composed on the basis of the standards of EN 1993-1-5, DASt-R015, and DIN 18800, and the optimum program was coded in accordance with the standards based on Real-Coded Genetic Algorithms. The genetic operators for the developed program resulted in a stable solution with crossover probability between 12.5 and 50\%, and the perturbation vector for outbreeding could obtain the best result with the model being applied of feasible design variable space of $20-30 \%$. Additionally, the increase of yield strength resulted in decreased value of the objective function, and it was found through the change of the value of the constraint function that the thickness of web was an important factor in the optimum structural design.
\end{abstract}

\section{Introduction}

Plate girders reinforced with stiffeners are widely used for long-span steel structures and under condition of heavy load. Nonetheless, the thin and long webs are fundamentally subjected to buckling and corrugated webs surfaced as a solution to this problem. Corrugated plates were actively applied in various fields starting with the patent granting to Palmer Ltd. of Great Britain in 1829, and the application of corrugated plates is easily seen even now in such structures as container, train, and hangar gate. The application of corrugation to ordinary structures started with webs on the basis of aeronautic findings in 1924, and it has been used in ordinary steel structures since early 1960s [1].

Technological achievements of corrugation in Europe are well explained in the standards of EN 1993-1-5 [2], DIN 18800 T1-3 [3], and DASt-R015 [4] more fundamentally than those of the USA or Japan because of its earlier commercialization in Europe. The structural design mechanism is such that the corrugated web is designed to withstand shear force only because the bending moment is not transferred on the web owing to the accordion effect of the corrugated web, and the design scheme for plate girder is also similar [5]. Corrugation has been mainly designed for trapezoidal or sinusoidal shape [6]. The stability of shear buckling due to thinness is affected by the height and wave form of the web, and local buckling, global buckling, and their coupling take place. Investigations of corrugated web mainly focused on shear buckling behavior and safety, elastic transverse buckling due to bending of corrugated web girder, behavior of fatigue load and improvement of the fatigue life, and so on [7-12]. Particularly, the initial imperfection is adequately sensitive to bring about shear buckling, and verification of the buckling performance for the safety becomes a very important factor.

Easley and McFarland [13] proposed an appropriate solution to explain the overall buckling with orthotropic 
theory in their investigation of shear buckling and verified it through an experiment, and later Elgaaly and Dagher [14] published their research findings on the history and development of corrugated web research. Additionally, Abbas et al. [15] analytically investigated the characteristics of interactive buckling and constraint and dealt with the influence of initial imperfection. Driver et al. [16] proposed a design range of shear buckling of corrugated web bridge, and Moon et al. [17] presented shear strength that could provide better evaluation. Circular corrugation was investigated by Chan et al. [18] for accurate analysis and the improvement of stiffness owing to the corrugation in comparison to smooth web was compared in terms of analytical and experimental results. Vertical and horizontal circular corrugation shape were also investigated to the elastic-plastic zone.

Zhang et al. [19] started a research to define the optimum section for hot-rolled wholly corrugated web (WCW) beam. Particularly, they developed an optimum parameter set for WCW H-shaped beam based on the optimization of plate web beam and investigated the influence of corrugation parameters. They cited research findings of Li et al. [20] to explain that the buckling resistance capacity of the corrugated web beam against axial force was 1.5 to 2 times greater than plate web beam. The equation for equivalent buckling strength was used as dimensionless objective function with cross-sectional area and section height. However, the depth of corrugation wave was restricted to between 2 and 4.6 times the thickness of corrugation web as a boundary condition, and this restriction considered general conditions for the production of hot-rolled WCW $\mathrm{H}$-shaped web. When corrugation web strip is fabricated by welding onto flange, the range of the restriction on the cross section is different in reality [21]. Additionally, the resistance capacity of thin corrugated web against shear buckling becomes the design main factor, and the research on the optimum structural design in consideration of this factor and the search techniques is still insufficient.

This study proposes a method for an optimum structural design of sinusoidal corrugated web (SCW) beam and aims at developing its global optimization program. The objective function is the minimum weight, and the constraint functions are applied pursuant to standards of EN 1993-1-5 [2], DIN 18800 T1-3 [3], and DASt-R015 [4]. The design program is developed by applying a Real-Coded Genetic Algorithm (RCGA), which does not require decoding, and is applied to an exemplar case of simple supported beam to investigate the variation of solutions with respect to the parameters of the genetic operator and the application characteristics. This paper is organized as follows. First section introduces the direction and purpose of this study, and the second section describes the design method for the SCW beam to be applied in this study. Section 3 describes the mathematical model for minimum weight design and explains the developed program. Finally, Section 4 carries out the analysis of the results of example, and the conclusion of the optimum design is discussed in Section 5.



FIGURE 1: Shape of sinusoidal corrugation with the shape parameters.

\section{Design Standards of SCW (Sinusoidal Corrugated Web) Beam}

In the design of SCW beam, there are two important characteristics of structural design mechanism. One is that the functions of web and flange are separately applied, and the other is the resistance against shear buckling is very complex for the analysis. Particularly, the number of design variables of web to determine the shear performance is very large, and the height of the web still affects the resistance of flange against the bending moment even if the roles of flange and web are separated.

2.1. Shape and Shear Buckling Stress of SCW. The shape parameters for the design of sinusoidal corrugated web can be defined by the depth of corrugation wave $\left(a_{3} / 2\right)$, weigh length $(w)$, and web thickness $\left(t_{w}\right)$ as shown in Figure 1. The corrugation function of sinusoidal shape $(y)$, half-wave length $(s)$, and inertia moment of corrugated web $\left(I_{x}\right)$ can be defined as shown in (1) (3), respectively.

$$
\begin{aligned}
y & =\frac{a_{3}}{2} \sin \left(\frac{\pi}{w} x\right) \\
s & =\int_{0}^{w} \sqrt{1+\left\{\frac{a_{3} \pi}{2 w} \cos \left(\frac{\pi}{w} x\right)\right\}^{2}} d x, \\
I_{x} & =\int_{0}^{w}\left[\frac{t_{w}^{3}}{12}+t_{w}\left\{\frac{a_{3}}{2} \sin \left(\frac{\pi}{w} x\right)\right\}^{2}\right] d x .
\end{aligned}
$$

Shear buckling of a web is different from a plate girder in that a web does not need stiffeners due to its resistance to corrugation, and a thin corrugated web can perform as good as a thick flat web. However, corrugation webs have to secure stability against local buckling occurring in one corrugation, global buckling occurring in several corrugations, or interactive buckling, and EN 1993-1-5 Annex D specifies the idealized critical shear stress as shown in (4a) and (4b). Pasternak and Hannebauer [1] claimed that sinusoidal corrugation resulted in better than trapezoidal corrugation owing to its smoother shape and introduced theoretical equations based on global buckling experiments as shown in (5) [21].

$$
\begin{aligned}
\tau_{\mathrm{cr}, l} & =\left(5.34+\frac{a_{3} s}{h_{w} t_{w}}\right) \frac{\pi^{2} E}{12\left(1-v^{2}\right)}\left(\frac{t_{w}}{s}\right)^{2}, \\
\tau_{\mathrm{cr}, g} & =\frac{32.4}{t_{w} h_{w}^{2}} \sqrt[4]{D_{x} D_{y}^{3}},
\end{aligned}
$$




$$
\begin{aligned}
& \tau_{\mathrm{cr}, \boldsymbol{g}} \\
& \quad=\left\{15.065+\left(\frac{w a_{3}}{h_{w} t_{w}}\right)^{2}\right\}\left\{t_{w}\left(\frac{h_{w}}{2}\right)^{2}\right\}^{-1} \sqrt[4]{D_{x} D_{y}^{3}}
\end{aligned}
$$

2.2. Shear Resistance Capacity of SCW. The web against shear force should be designed by (6a) and (6b), and the factors $C$ and $\kappa$ have different specified values depending on EN 1993-15 [2] or DASt-R015 [4]. Nonetheless, the basic design concept is the same, and the reduction factor of $\kappa$ is determined by limit slenderness ratio with respect to local and global buckling.

$$
\begin{aligned}
& V_{S d} \leq V_{R d}\left(=\frac{V_{R k}}{\gamma_{M}}\right), \\
& V_{R k}=C \cdot \kappa \cdot f_{y, w} \cdot h_{w} \cdot t_{w} .
\end{aligned}
$$

EN 1993-1-5 [2] and Pasternak and Hannebauer [1] defined the value of $C$ as $1 / \sqrt{3}$, and DASt-R015 [4] defined it as 0.35. Additionally, reduction factors of DASt-R015 [4] are presented differently depending on the buckling type as (7), and the design realm for the resistance to the shear force also differs a little. DASt-R.015 [4] standard sets the lower slenderness ratio $\left(\lambda_{c}\right)$ for local or global buckling as the reduction factor. Particularly, global buckling stress is specified in the range between 0.5 and 2 times local buckling stress.

$$
\kappa=\frac{0.84}{\lambda_{c}} \leq 1.0,
$$

where

$$
\begin{aligned}
\lambda_{c} & =\max \left(\lambda_{c, l}: \lambda_{c, g}\right), \\
\lambda_{c, l} & =\sqrt{\frac{f_{y, w}}{\sqrt{3} \tau_{\mathrm{cr}, l}},} \\
\lambda_{c, g} & = \begin{cases}\sqrt{\frac{2 f_{y, w}}{\sqrt{3} \tau_{\mathrm{cr}, g}}} & 0.5 \leq \frac{\tau_{\mathrm{cr}, g}}{\tau_{\mathrm{cr}, l}} \leq 2.0 \\
\sqrt{\frac{f_{y, w}}{\sqrt{3} \tau_{\mathrm{cr}, g}}} & \text { other. }\end{cases}
\end{aligned}
$$

Although EN 1993-1-5 [1] standard is also similar to other standards, the equation for reduction factor is proposed differently for local buckling and global buckling as shown in (9a) and (9b). However, the same equation for slenderness ratio is used for local buckling and global buckling.

$$
\begin{gathered}
\kappa_{l}=\frac{1.15}{0.9+\lambda_{c, l}} \leq 1.0, \\
\kappa_{g}=\frac{1.5}{0.5+\lambda_{c, g}^{2}} \leq 1.0,
\end{gathered}
$$

where

$$
\lambda_{c, l(g)}=\sqrt{\frac{f_{y, w}}{\sqrt{3} \tau_{\mathrm{cr}, l(g)}}} .
$$

2.3. Resistance Capacity of Flange. When moment and shear force are designed to resist flange and web, respectively, according to the design methods of Siokola [5] and Pasternak and Hannebauer [1], the flange should be designed by (11a) and (11b), where $N_{S d}$ is the axial force of the flange member, $N_{R d}$ is the design stress, and $\gamma_{M}$ is the partial safety factor.

$$
\begin{aligned}
& N_{S d} \leq N_{R d}\left(=\frac{N_{R k}}{\gamma_{M}}\right), \\
& N_{R k}=\min \left(N_{Z, R k}: N_{D, R k, l}: N_{D, R k, g}\right) .
\end{aligned}
$$

The computation of axial force of flange depends on whether it is tensile force or compressive force, and the design resistance capacity of flange against tensile force is given by the following equation:

$$
N_{Z, R k}=f_{y} \cdot b_{f} \cdot t_{f}
$$

Safety against buckling should be considered in case of compression, and both local and global buckling should be examined. First of all, local buckling should be examined for the limit width-thickness ratio, and the reduced stress of flange by applying the effective width of flange reduced by corrugation is computed by (13), where $\sigma_{1}$ is the largest value of compressive stress [3].

$$
N_{D, R k, l}=\sigma_{1} \cdot b_{f} \cdot t_{f} .
$$

Global buckling of flange is affected by the spacing of the lateral support and is defined by (14) [1], where $k_{c}$ is specified in DIN 18800 T.1 Tab.8 [3] and $c$ is the lateral support length of flange. However, if the load exerted on the flange does not surpass the maximum load, the safety examination can be omitted.

$$
N_{D, R k, g}=\frac{0.5 \pi}{\sqrt{12}} \sqrt{E f_{y}} \frac{b_{f}^{2} t_{f}}{k_{c} c} .
$$

Additionally, design issues of various combination stress and concentrated load need to be considered. Moreover, constraint on the shear force and moment combination stress can result in reduction of the load capacity mainly when shear force and flexural moment take place simultaneously at the end of a continuous beam [1].

\section{Optimum Structural Design by RCGA (Real-Coded Genetic Algorithms)}

The difficulty of optimization problems has been addressed with numerical analytic methods and development of computer to make the optimization problem easier. Among these developments, GA is easy to explain and simple to program. Although it has been applied to many problems, the behavior can be very complex and it is not easy to predict how it operates and what kind of operators are most appropriate. Nevertheless, it is a very useful technique to be applied to global optimization problems or problems which are difficult to be dealt with by conventional methods. 
3.1. Formulation of an Optimization Problem. The optimum structural design refers to the method to simultaneously satisfy the constraints imposed on the design variables and to compute the design variables, which minimizes the objective function. The economic design problem usually entails the weight of the member as the objective function. Thus, the minimum weight design problems with objective function $F(\mathbf{x})$ can be defined as (15a) and (15b), and it is the goal to search for the design variable of minimum weight, which satisfies the constraints. Since the unit weight shown in the objective function is a constant in case of all members having the same materials, it follows that minimum volume or crosssectional area means also minimum weight.

$$
\begin{array}{ll}
\text { mininize } & F(\mathbf{x})=\sum_{i=1}^{n e} \rho_{i} A(\mathbf{x})_{i} L_{i} \\
\text { subject to } & g(\mathbf{x})_{j} \leq 0 \quad(j=1, \ldots, n) \\
& \mathbf{x}^{l} \leq \mathbf{x} \leq \mathbf{x}^{u},
\end{array}
$$

where

$$
A(\mathbf{x})_{i}=\left\{2 b_{f} t_{f}+h_{w} t_{w} \frac{s}{w}\right\}_{i}
$$

The constraint functions are composed of constraints of slenderness ratio $\left(g(\mathbf{x})_{1}, g(\mathbf{x})_{2}\right)$, internal forces $\left(g(\mathbf{x})_{3}, g(\mathbf{x})_{4}\right)$, and deflection $\left(g(\mathbf{x})_{5}\right)$ as shown in (17a), (17b), (17c), (17d), and (17e). The constraints on slenderness ratio are specified in El.120 of DASt_R015, K115, and El.409 $[1,5]$.

$$
\begin{aligned}
& g(\mathbf{x})_{1}=\frac{\lambda_{p, w}}{\lambda_{p, w, \max }}-1.0 \leq 0.0, \\
& g(\mathbf{x})_{2}=\frac{\lambda_{p, f}}{\lambda_{p, f, \max }}-1.0 \leq 0.0, \\
& g(\mathbf{x})_{3}=\frac{V_{S d}}{V_{R d}}-1.0 \leq 0.0 \\
& g(\mathbf{x})_{4}=\frac{N_{S d}}{N_{R d}}-1.0 \leq 0.0 \\
& g(\mathbf{x})_{5}=\frac{\delta_{\text {max }}}{\delta_{\text {limit }}}-1.0 \leq 0.0
\end{aligned}
$$

where

$$
\begin{aligned}
\lambda_{p, w} & =0.8\left(\frac{h_{w}}{t_{w}}\right) \sqrt{\frac{f_{y, w}}{\kappa_{\mathrm{cr}, l} E}}, \\
\lambda_{p, w, \max } & =0.316 \sqrt{\frac{E}{f_{y, w}}}, \\
\lambda_{p, f} & =\frac{b_{f}+a_{3}}{t_{f}}, \\
\lambda_{p, f, \max } & =1.03 \sqrt{\frac{E}{f_{y, f}}} .
\end{aligned}
$$

3.2. Real-Coded Genetic Algorithm (RCGA). This study selected RCGA to obtain the solution for an optimization, and this algorithm uses not a binary number but a real number to represent the genetic information. In designing the minimum weight for steel structures, the design variables generally indicate the cross-sectional area of the structural members. Even with the objective function in (15a) and (15b), the parameters of the cross-sectional shape of a single member with constant weight are assigned to the design variables. The structural members mainly use $\mathrm{H}$-shape section steel, channels (C-shape section), and square-shape steel pipes, and the design variables increase as the shape of these members becomes complex, and the number of members increases. Therefore, it is difficult and inefficient to express the increasing design variables in genetic information in a usual binarycoded GA (BCGA). In case of SCW beams, which have complex shapes, it is more practical and reasonable to use RCGS that use an actually used variable as the design variable rather than the complicated BCGA to solve the weight optimization problem. The GA like simulated annealing (SA) can be applied to various types of problems, and this is a global optimization method widely used. This method emulates the genetics of nature to search for an appropriate solution by the process of artificially creating new operators for next generation through the operations of selection, crossover, and mutation of a design variable set, which possess similar structure as the biological genetic factor, that is, chromosomes [22]. Thus, unlike existing optimization methods, which search for local solution and then repeat the computation with several initial points to search for the global optimum, GA carries out a probabilistic search with not a design point but a design group. Thus, it is evaluated to have higher reliability to reach the global optimum. RCGA has the same algorithm as GA with binary numbers. However, it is composed of real numbers not binary numbers so that decoding is not required, and it differs a little from GA in operation, type, and method.

Genetic operation between parent generation and offspring generation inherits the trait by using fitness in the process of searching for the solution. Fitness in the context of GA means the scale of capacity to survive in ecological system, and it determines whether copying of the genetic information of each individual in the mating pool is allowed or not. Therefore, the individual with high fitness means higher probability of being selected as the parent of next generation. The constrained optimization problem of this paper consisted of an objective function and the unequal constraints. As shown in (15a) and (15b), the objective function is the weight of a structure, and constraints are design codes for SCW beams. This minimization problem can be converted by a series of unconstrained minimization problems as

$$
\begin{gathered}
\min \quad \phi_{k}(\mathbf{x})=F(\mathbf{x})+r_{p} P(\mathbf{x}) \\
P(\mathbf{x})=\sum_{i=1}^{n} \min \left\{0, g(\mathbf{x})_{i}\right\}^{q},
\end{gathered}
$$

where $\phi_{k}(\mathbf{x})$ is fitness function, $P(\mathbf{x})$ is a penalty function, $g(\mathbf{x})_{i}$ is $i$ th constraint function, and $r_{p}, q(=2)$ are the penalty coefficients and the multiplier for the penalty function, 
respectively. To intuitively express the fitness values, the minimization problem of (19a) can be expressed as a maximization problem of the fitness function $\phi(\mathbf{x})$, as shown in

$$
\max \phi(\mathbf{x})=\frac{c_{2}}{c_{1}+\phi_{k}(\mathbf{x})},
$$

where $c_{1}$ and $c_{2}$ are the constants.

The offspring generation $\mathbf{x}^{\text {offspring }}$ is formed from parent generation $\mathbf{x}^{\text {parent }}$ through such operations as selection, crossover, mutation, and so forth, and each operation is carried out with the purpose of increasing fitness or maintaining superior traits. Particularly, since the probabilistic variables of genetic operation have different effective values depending on mathematical models, it is important to select the appropriate variable. The genetic operations selected for this study are crossover, mutation, elitist strategy, and outbreeding which are explained as follows.

Crossover of binary variables refers to mixing of genetic information from the chromosomes of parent generation and involves one point, several points, homogeneous crossover, and so forth. However, RCGA does not convert the genetic information into chromosomal form with genetic trait, and its crossover methods (interpolation, extrapolation, heuristic, hybrid heuristic with crossover, and so forth) are different. Interpolation/extrapolation and heuristic methods follow the process expressed in (21a) and (21b) to mix and inherit the genetic information, where $r$ is a random number.

$$
\begin{aligned}
& \mathbf{x}^{\text {offspring }}= \pm r \mathbf{x}_{i}^{\text {parent }}+(1 \mp r) \mathbf{x}_{j}^{\text {parent }}, \\
& \mathbf{x}^{\text {offspring }}=\mathbf{x}_{i}^{\text {parent }}+r\left(\mathbf{x}_{i}^{\text {parent }}-\mathbf{x}_{j}^{\text {parent }}\right) .
\end{aligned}
$$

Mutation like crossover means the genetic information of offspring is defined by a probability different from parent. Since binary variable has the values of 0 and 1 only, its mutation is easily carried out by changing 0 to 1 and vice versa. However, real number variable makes the movement to new search space of more wide design range to escape from local minimum. It results in a mutation probability relatively higher than binary variable to be applied differently depending on the problems and is defined as shown in

$$
x_{i}^{\text {new }}= \begin{cases}x_{i}^{\text {low }}+r\left(x_{i}^{\text {high }}-x_{i}^{\text {low }}\right), & \text { if } r \leq p_{M} \\ x_{i}^{\text {current }}, & \text { if } r>p_{M} .\end{cases}
$$

Elitist strategy keeps the best population forcefully in order to prevent it from being excluded or changed by crossover or mutation in the process of selecting each next generation. It can maintain the best fitness by preserving the best individual with the overall increase in the fitness during the process of changing the generation [23].

Lastly, outbreeding replaces some part of a population with new population at each generation in order to prevent the lowering of search efficiency in the design space due to similar genetic information or occurrence of homogeneous individual with each new generation. This method applies

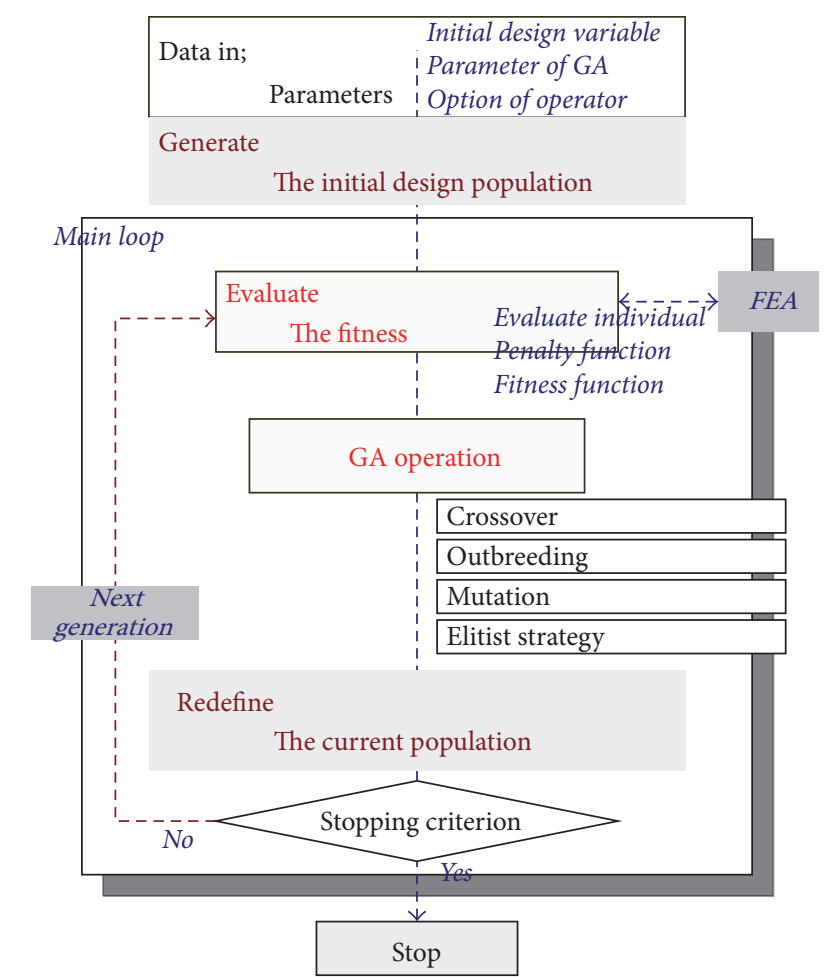

FIGURE 2: Flowchart for the optimum design programing with RCGA.

preset perturbation vector $d \mathbf{x}$ and random number vector $\mathbf{r}$ to existing population and is defined as shown in [24]

$$
\mathbf{x}^{\text {new }}=\mathbf{x}^{\text {current }} \pm \mathbf{r} \cdot \operatorname{diag}(d \mathbf{x}) .
$$

Since perturbation vector is generally determined at the early stage of the genetic operation, it can be defined by the size of the design variable space, and it affects convergence of the solution and its accuracy.

\subsection{Flowchart of the Developed Optimum Design Program.}

This study used RCGA for the optimum structural design of SCW beam, and this program searches the global minimum by implementing the aforementioned genetic operation. The flowchart for the developed program is shown in Figure 2.

In calculating the fitness of the flowchart in Figure 2, $V_{S d}, N_{S d}$, and $\delta$ in (17a), (17b), (17c), (17d), and (17e) were member stresses and deflection and can be obtained by a structural analysis. While it is easy to obtain the solution for a simple beam, it is difficult to find those for a complex one. Particularly, for tapered beams under various external force or steel structures with many members, it is more practical and useful to obtain an approximate solution using a finite element analysis (FEA). The stopping rule in metaheuristic methods like SA, GA, or RCGA is generally determined by either the mean fitness convergence of the population, the best fitness convergence, or maximum generation number. This paper compared the convergence process of the fitness by setting maximum generation number to a specified value in order to determine the probabilistic parameter of RCGA 


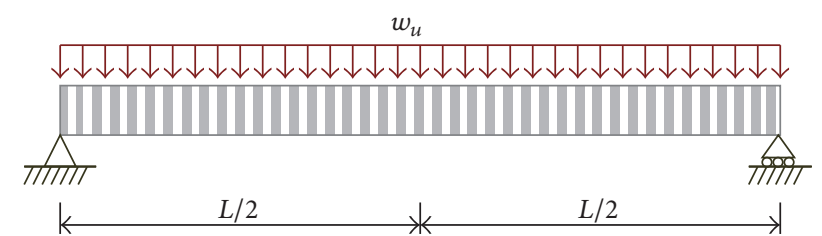

Figure 3: Shape and external load condition of the simple beam model.

and the number of individuals that are suitable for the optimal design of SCW beam.

\section{Optimum Structural Design of Simple Supported SCW Beam}

RCGA is applied to compute the global minimum for a problem consisting of constraints as well as the objective function to minimize the cross-sectional area of SCW beam. The efficiency of the probabilistic parameters is examined through the analysis applied to the simple supported beam as shown in Figure 3.

RCGA generates the offspring from the parent through various genetic operations and can obtain the generation with better fitness as the number of generations increases. Many parts of the operations in this process are to generate a random number and to determine the genetic operator. The genetic operators are carried out by the random number through the probability of the operators. The probabilistic parameters of the operators are crossover probability $p_{C}$ and mutation probability $p_{M}$. Mutation is to escape from local minimum to search for the global minimum. Outbreeding generates another parent through the imposition of perturbation vector and increases the search efficiency, which was lowered due to the selection of similar traits of the parent, in the design space. In other words, the size of perturbation vector space for enlarging the design space affects the search efficiency. Thus, this section discusses the convergence and efficiency of the solution of the program in response to the change of probabilistic variables and perturbation vector, when they were applied to the SCW beam for its optimum design.

The adopted model has a span $L$ of $10 \mathrm{~m}$ and uniform load of $w_{u}=100 \mathrm{~N} / \mathrm{m}$ is applied. The elastic modulus $E$, Poisson ratio $v$, and yield stresses $f_{y}$ are $210 \mathrm{GPa}, 0.3$, and $240 \mathrm{MPa}$, respectively. The shape of the corrugation is set to be $a_{3}=$ $4.0 \mathrm{~cm}$ and $w=7.75 \mathrm{~cm}$. The sinusoidal wave selected for this study was generated by an automatic machine developed by Zeman \& CO of Vienna, Austria [5, 25]. The deflection limit was set to be $\delta_{\text {limit }}=L / 300$, and the design force used the maximum bending moment and maximum shear force and DASt-R015 was applied to the design.

4.1. Convergence in Response to the Increase in the Numbers of Generations and Populations. GA generally improves the fitness with the increase in the numbers of populations and generations. Although good solutions can be obtained even with some changes of the parameters due to the fact
TABLE 1: Result of optimum structural design by RCGA $\left(p_{C}=0.875\right.$; $\left.p_{M}=0.3 ; d \mathbf{x}=0.001 \mathbf{x}^{d}\right)$.

\begin{tabular}{lcccccc}
\hline $\begin{array}{l}\text { Number of } \\
\text { populations }\end{array}$ & $\begin{array}{c}F(\mathbf{x}) \\
\left(\mathrm{cm}^{2}\right)\end{array}$ & $b_{f}(\mathrm{~cm})$ & $\begin{array}{c}t_{f} \\
(\mathrm{~mm})\end{array}$ & $h_{w}(\mathrm{~cm})$ & $\begin{array}{c}t_{w} \\
(\mathrm{~mm})\end{array}$ & $P(\mathbf{x})$ \\
\hline 10 & 157.896 & 21.77 & 15.0 & 173.70 & 4.6 & 0.0 \\
20 & 159.803 & 24.05 & 17.4 & 135.95 & 4.9 & 0.0 \\
30 & 160.560 & 32.41 & 13.2 & 141.76 & 4.6 & 0.0 \\
40 & 157.858 & 31.32 & 12.7 & 151.64 & 4.5 & 0.0 \\
50 & 154.459 & 18.57 & 20.4 & 150.51 & 4.6 & 0.0 \\
60 & 156.806 & 24.93 & 14.8 & 157.99 & 4.6 & 0.0 \\
70 & 158.920 & 20.76 & 17.4 & 157.39 & 4.8 & 0.0 \\
80 & 157.674 & 21.24 & 18.2 & 151.43 & 4.6 & 0.0 \\
90 & 157.429 & 29.29 & 13.2 & 153.16 & 4.5 & 0.0 \\
100 & 153.837 & 25.83 & 14.1 & 157.73 & 4.5 & 0.0 \\
\hline
\end{tabular}

TABLE 2: Result of optimum structural design by RCGA $\left(p_{C}=0.25\right.$; $\left.p_{M}=0.0 ; d \mathbf{x}=0.3 \mathbf{x}^{d}\right)$.

\begin{tabular}{lcccccc}
\hline $\begin{array}{l}\text { Number of } \\
\text { populations }\end{array}$ & $\begin{array}{c}F(\mathbf{x}) \\
\left(\mathrm{cm}^{2}\right)\end{array}$ & $b_{f}(\mathrm{~cm})$ & $\begin{array}{c}t_{f} \\
(\mathrm{~mm})\end{array}$ & $h_{w}(\mathrm{~cm})$ & $\begin{array}{c}t_{w} \\
(\mathrm{~mm})\end{array}$ & $P(\mathbf{x})$ \\
\hline 10 & 164.581 & 18.47 & 18.7 & 167.67 & 5.0 & 0.0 \\
20 & 153.870 & 24.07 & 16.3 & 143.82 & 4.6 & 0.0 \\
30 & 154.036 & 20.05 & 19.0 & 149.97 & 4.5 & 0.0 \\
40 & 153.161 & 23.73 & 14.9 & 160.15 & 4.5 & 0.0 \\
50 & 152.794 & 29.02 & 13.1 & 149.03 & 4.5 & 0.0 \\
60 & 154.124 & 22.97 & 16.2 & 153.78 & 4.5 & 0.0 \\
70 & 154.037 & 25.49 & 13.4 & 166.65 & 4.5 & 0.0 \\
80 & 152.831 & 20.36 & 18.1 & 153.92 & 4.5 & 0.0 \\
90 & 152.959 & 26.14 & 14.3 & 151.65 & 4.5 & 0.0 \\
100 & 153.139 & 24.60 & 15.3 & 150.77 & 4.5 & 0.0 \\
\hline
\end{tabular}

that they are based on probabilistic operation, satisfactory results in proportion to the quantity of computation are not guaranteed. Thus, in this section, we investigate the optimum results and fitness for the model in response to the increase in the numbers of generations and populations. This study increased the number of populations from 10 to 100 in increments of 10 and the number of generation up to 1000 .

Figures 4 and 5 show the change of objective function and fitness based on the analysis result for the model by the elite individual with crossover probability $p_{C}$, mutation probability $p_{M}$, and perturbation vector $d \mathbf{x}$ for outbreeding (\% of the total design space) of $87.5 \%, 30 \%$, and $0.1 \%$ and $25 \%$, $0 \%$ (no mutation), and 30\%, respectively.

Tables 1 and 2 show the values of design variables and penalty function for each number of converged elite based on Figures 4 and 5. The value of penalty function not exceeding zero means that the objective functions with the design variables are within the effective range not violating the constraints. These two results intuitively indicate that the increase in the number of generations results in convergence but that the convergence speed is slowed down with the decrease in the number of populations. Additionally, the final convergence value with the simple increase in the population does 


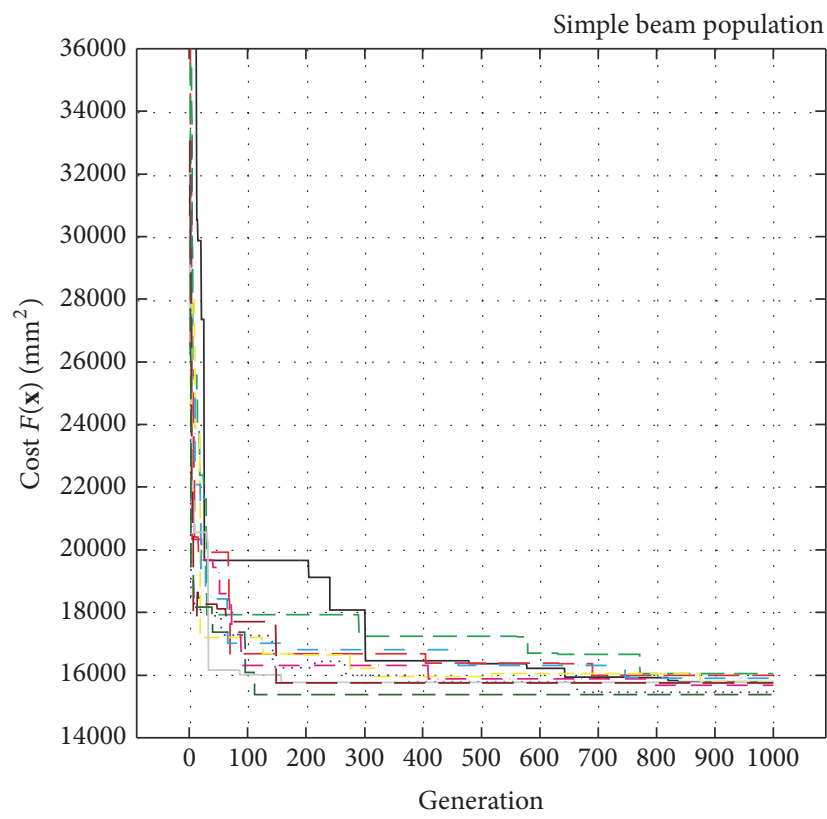

- 10 populations

- - 20 populations

- - 30 populations

40 populations

…. 50 populations

(a)

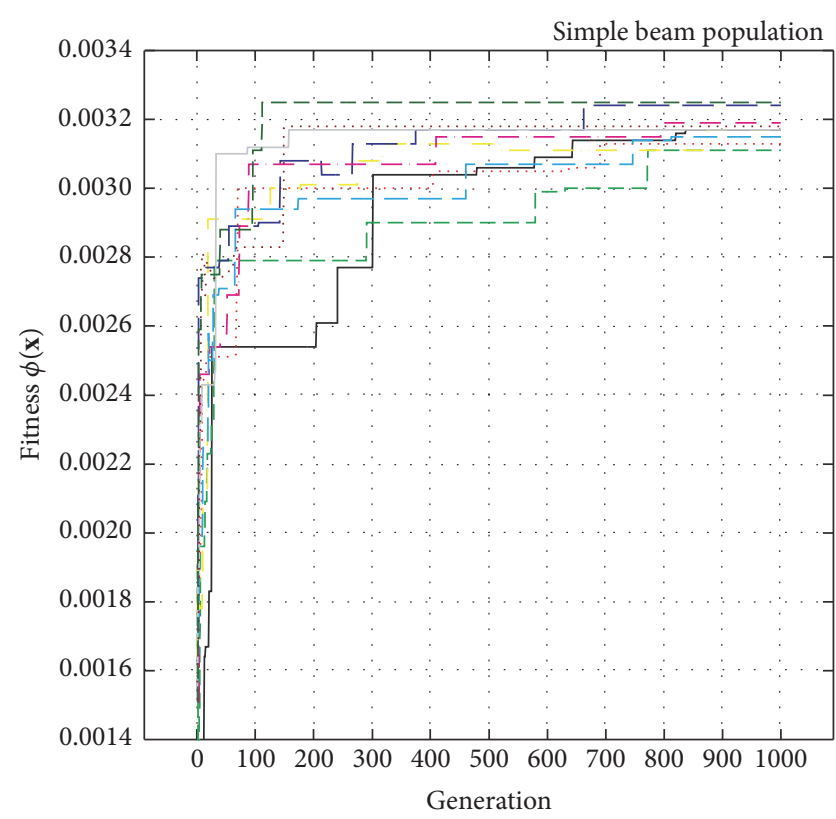

- 10 populations

…2 20 populations

--30 populations

- 40 populations

- - - 50 populations

(b)

FIGURE 4: Results and convergence of optimum design analysis $\left(p_{C}=0.875 ; p_{M}=0.3 ; d \mathbf{x}=0.001 \mathbf{x}^{d}\right)$. (a) Objective function. (b) Fitness.

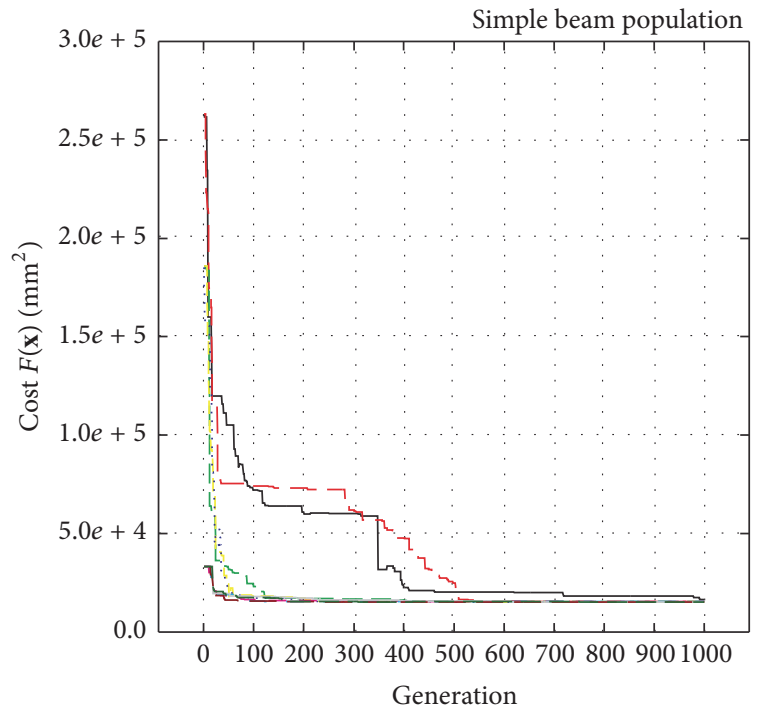

$\begin{array}{ll}-10 \text { populations } & \cdot--60 \text { populations } \\ ---20 \text { populations } & -\cdot-70 \text { populations } \\ ---30 \text { populations } & -80 \text { populations } \\ ---40 \text { populations } & ---90 \text { populations } \\ \ldots . .50 \text { populations } & ---100 \text { populations }\end{array}$

(a)

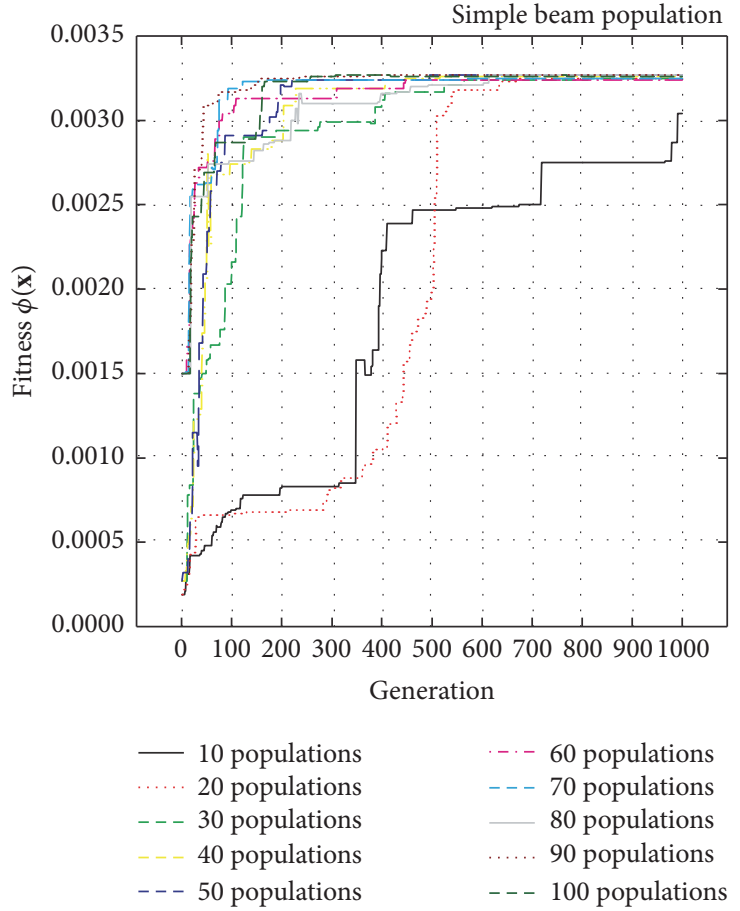

(b)

FIGURE 5: Results and convergence of optimum design analysis ( $\left.p_{C}=0.25 ; p_{M}=0.0 ; d \mathbf{x}=0.3 \mathbf{x}^{d}\right)$. (a) Objective function. (b) Fitness. 



FIGURE 6: Constraint function of converged population $\left(p_{C}=0.25\right.$; $\left.p_{M}=0.0 ; d \mathbf{x}=0.3 \mathbf{x}^{d}\right)$.

not result in increased fitness. Examining Tables 1 and 2, $t_{w}$ converged to $4.5 \mathrm{~mm}$, and the other design variables had a little difference in the final converged value.

The values of $P(\mathbf{x})$ shown in Tables 1 and 2 indicate that the elite converged with the increase in the number of generations are within the feasible design region. However, the values of constraint functions are not all within the allowed range at the best. Figure 6 depicts the values of constraint function of the finally converged elite based on the result of Figure 5. The values shown in Figure 6 indicate that the constraints on stress of web $g(\mathbf{x})_{3}$ and stress of flange $g(\mathbf{x})_{4}$ all reached the boundary limit. Although there was no significant change of the pattern with the increase in the number of populations, the analysis of the lowest number of elite showed that it did not reach the boundary of $g(\mathbf{x})_{3}$ and that the result of objective function was the highest.

\subsection{Convergence of Probability Parameters and Perturbation} Vector. $p_{C}, p_{M}$, and $d \mathbf{x}$ affect the convergence of solution and global search. Each parameter carries out crossover and mutation depending on the random number, and mutation and outbreeding are performed with a purpose of enhancing global search and improving the search inefficiency due to the similarity of the traits of parents. Nonetheless, the optimum parameter varies by the formulated problem, and simple increase or decrease of the variables results in efficient or effective search result. Thus, this section compares the results by the operators and searches for the operator and parameter appropriate for the SCW beam, which was formulated in Section 3. Accordingly, RCGA are carried out on 100 populations for 1000 generations with only two operators at a time to examine the efficiency of the three operators.

Figure 7 shows the result without mutation, and Figures 8(a) and 8(b) are the results without outbreeding and crossover, respectively. The vertical axis of Figure 7 is dimensionless with $F_{0}=15260.7$, and it is the value of the objective function for the best elite individual among the analysis results of the model. The figure indicates that an improvement on the convergence cannot be expected with simple increase or decrease of the parameters. The figure depicting the result of analysis without mutation operation also shows the stable zone of the optimum solution converged according to $p_{C}$. In other words, $p_{C}$ between $12.5 \%$ and $50 \%$ resulted in a relatively stable analysis result and was relatively less influenced by $d \mathbf{x}$. Figure 8 (a) shows the result of analysis without outbreeding operation. Although it does not exhibit a relatively constant pattern, the least deviation is observed with $p_{M}=$ $20 \%$. Additionally, the analysis without crossover as depicted in Figure 8(b) shows the least deviation and convergence to the highest fitness at $p_{M}=10 \%$. This is definitely higher than $p_{M}$ of $0.1 \sim 1 \%$, which is typically used in binary-coded GA.

Figure 9 shows the optimization solutions based on the analysis result shown in Figure 7 when $d \mathbf{x}$ has the values of $20 \%$ and $30 \%$. The figure shows that the RCGA converged the fastest with $p_{C}$ of $25 \%$ and $37.5 \%$.

4.3. Minimum Weight Design of SCW Beam. This section examines how the design characteristics optimally result in the increase of the load and the yield strength. The yield stresses $f_{y}$ being applied for the analyses were 240, 270, and $330 \mathrm{MPa}$. Above all, as shown in Figure 10, the increases of the load and the yield stress resulted in the increase of the optimum weight and the decrease of the optimum weight, respectively.

Figure 11 shows the changes of the design variable and constraint function with respect to the analysis results shown in Figure 10. The result showing the polygons represents the value of the change of design variable with respect to the optimum design result by the increase of the load, and the bar graph shows the change of the constraint function. Since the constraint functions $g(\mathbf{x})_{1}$ and $g(\mathbf{x})_{3}$ are the constraint on the slenderness ratio and shear stress for the web and the constraint functions $g(\mathbf{x})_{2}$ and $g(\mathbf{x})_{4}$ indicate the constraint on the slenderness ratio and shear stress for the flange, the design variables of relatively high relationship with each constraint and the graph were overlapped for the illustration. This figure shows that the design variable increasing with the increase of the load is $t_{w}$, and the constraint function $g(\mathbf{x})_{1}$ continued to decrease in response to the change of the design variable. Although the change of the constraint function $g(\mathbf{x})_{3}$ in response to the increase or decrease of $h_{w}$ showed similar responses, it shows the result of reaching the boundary of actual design feasibility and the reason for it is that the value of constraint function is almost near 0 . On the other hand, the thickness of the flange $t_{f}$ does not keep increasing with the change of $t_{w}$. Nonetheless, the change of $b_{f}$ and $g(\mathbf{x})_{4}$ is maintained at the boundary of design feasibility similar to the web case. This change signifies that although there is some difference in all cases of $f_{y}$, the pattern is relatively the same. This also means the change of $t_{w}$ among the design variables actively influences the optimum design and its influence on the objective function is significant. Additionally, the point of crossing of the increase and decrease of the flange thickness $t_{f}$ is manifested at the same location as the point of crossing of the increase and decrease of $h_{w}$ curve. This variation starts to be manifested from the values of $240 \mathrm{MPa}$ and $60 \mathrm{~N} / \mathrm{mm}$ for $f_{y}$ and $w_{u}$, respectively, and it is the most evident also 

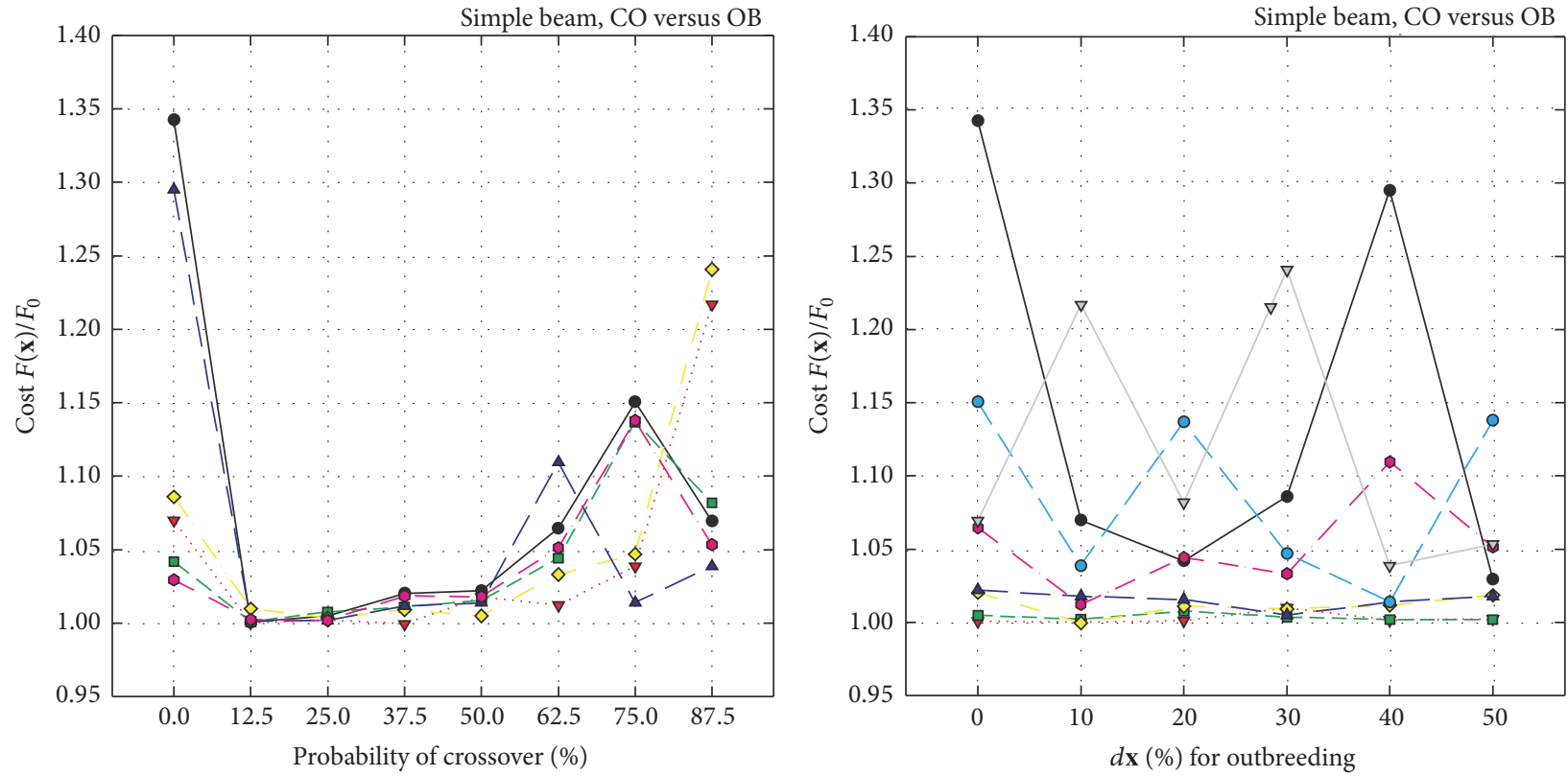

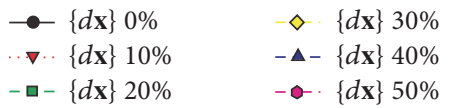

(a)

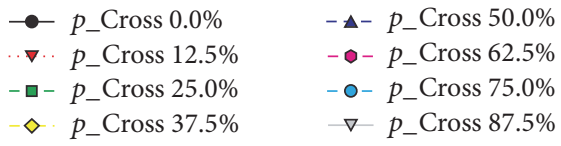

(b)

Figure 7: Analysis result by crossover and outbreeding operations. (a) Crossover. (b) Outbreeding.

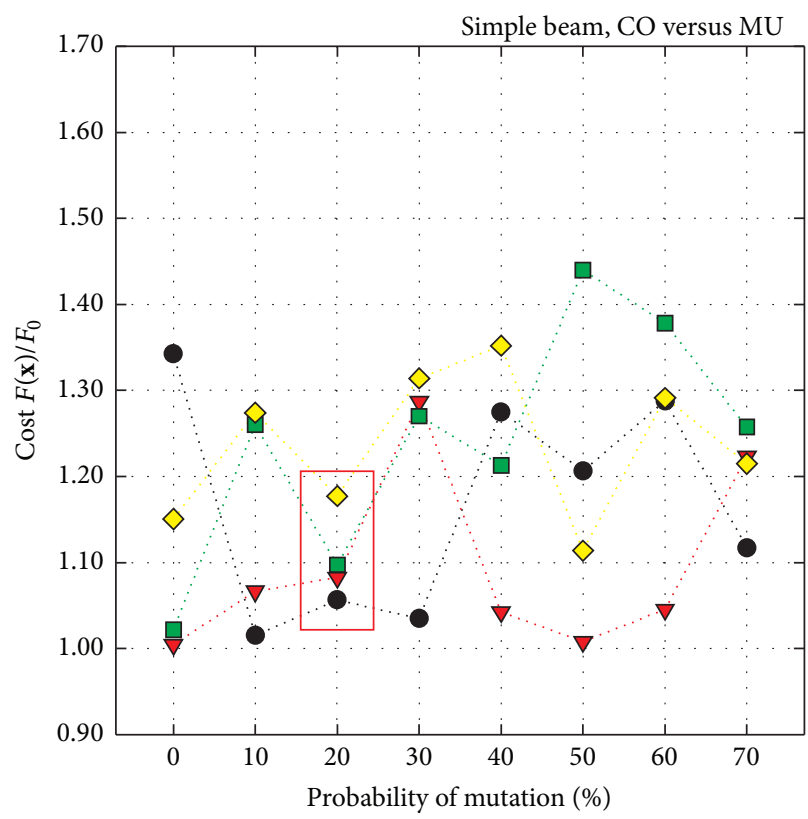

- P_Cross $0 \%$

$\nabla \cdot$ P_Cross $25 \%$

ㅁ. P_Cross 50\%

$\diamond$ p_Cross $75 \%$

(a)

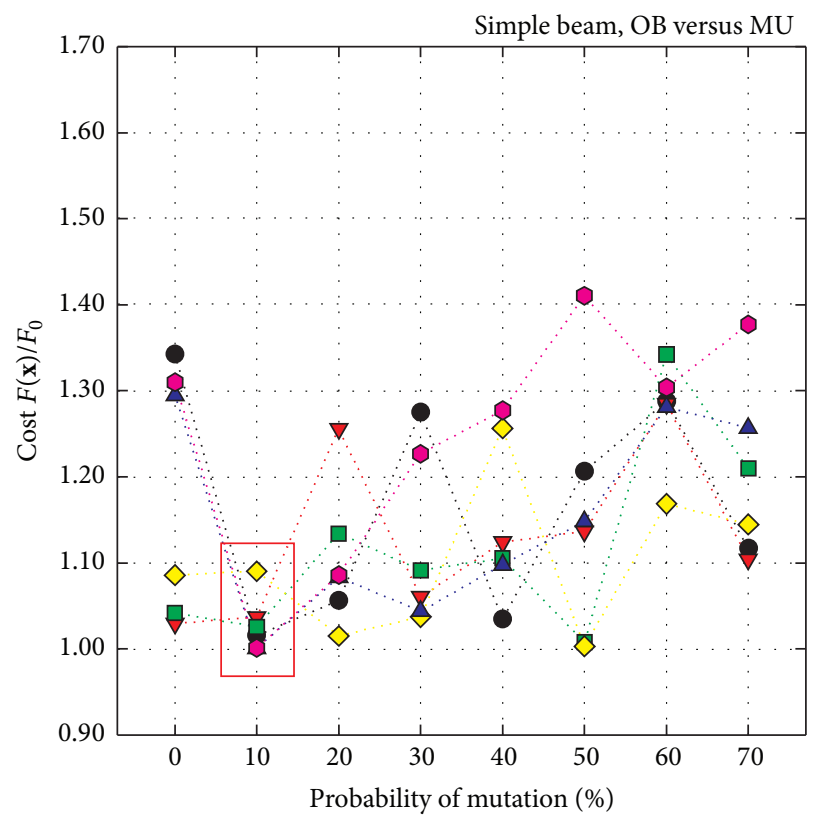

- - $\{d \mathbf{x}\} 0 \%$

$\nabla \cdot \cdot d \mathbf{x}\} 10 \%$

口.. $\{d \mathbf{x}\} 20 \%$ $\diamond \quad\{d \mathbf{x}\} 30 \%$

-.. $\{d \mathbf{x}\} 40 \%$

๑.. $\{d \mathbf{x}\} 50 \%$

(b)

FIGURE 8: Analysis result by mutation probability. (a) Mutation and crossover. (b) Mutation and outbreeding. 


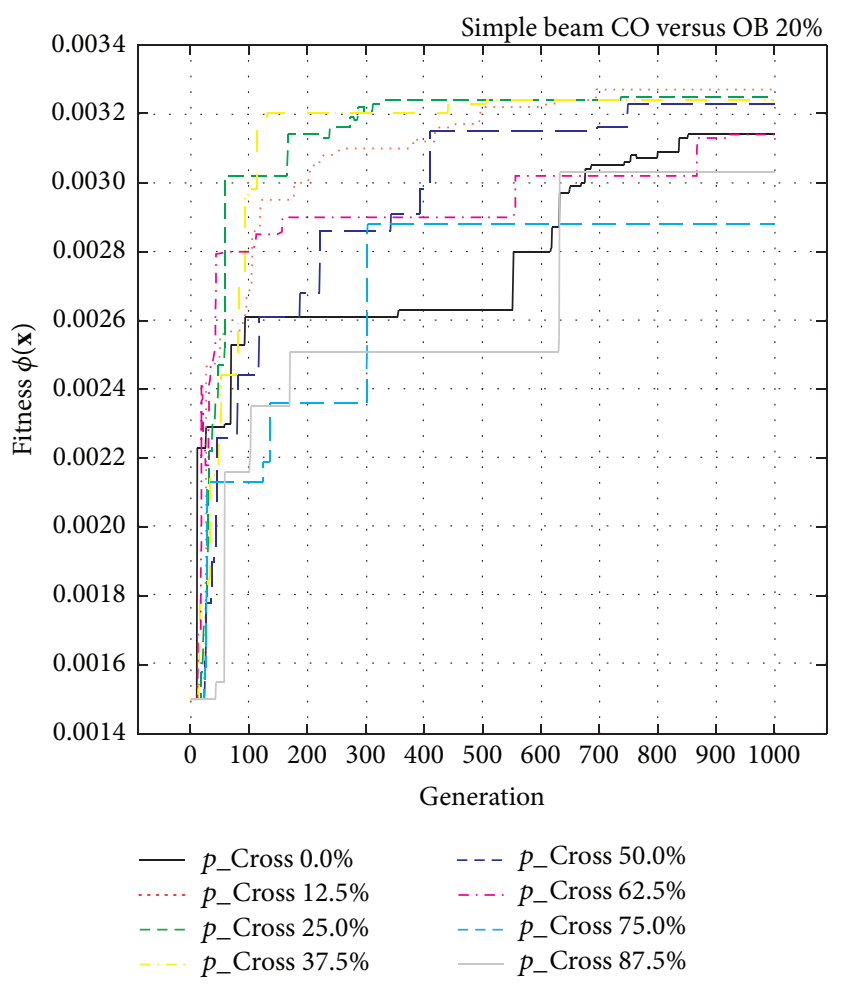

(a)



(b)

Figure 9: Result of optimum design analysis by crossover operation. (a) $p_{M}=0.0$ and $d \mathbf{x}=0.2 \mathbf{x}^{d}$. (b) $p_{M}=0.0$ and $d \mathbf{x}=0.3 \mathbf{x}^{d}$.

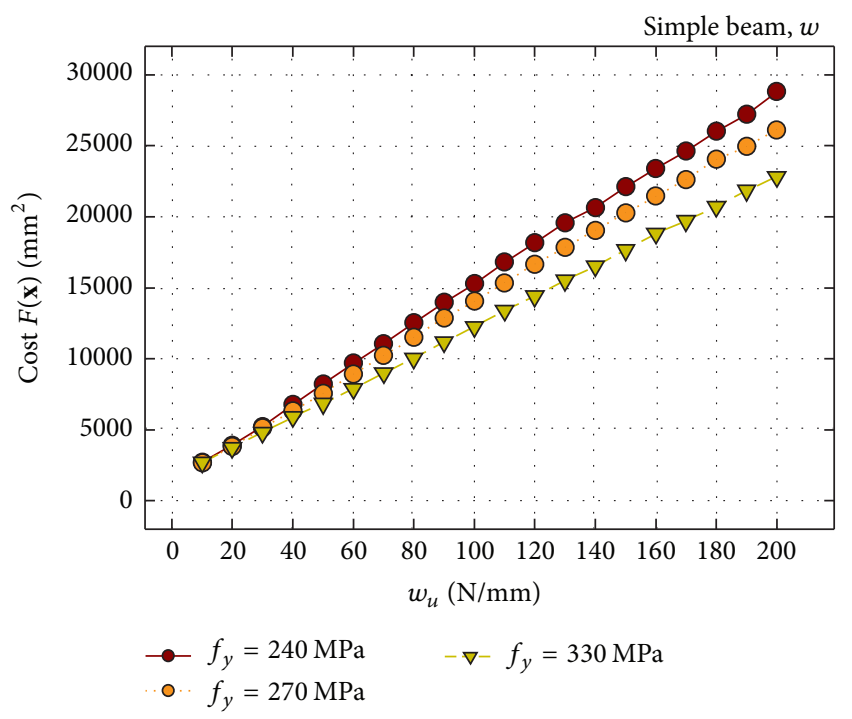

FIGURE 10: Variations of objective function by the yield strength.

for values of $f_{y}$ of $270 \mathrm{MPa}$ and $330 \mathrm{MPa}$ at the values of $w_{u}$ of $100 \mathrm{~N} / \mathrm{mm}$ and $130 \mathrm{~N} / \mathrm{mm}$, respectively. These results indicate that the design variables on the thickness are defined by slenderness ratio at each design boundary for the web and flange in response to the stress constraint. This is the result of the method of designing flange and web to resist the moment and shear force, respectively.
Finally, the case of fixed end of SCW beam is adopted and has a concentrated load and uniform load. The yield stresses $f_{y}$ being applied for the analyses were 240, 270, 300 , and $330 \mathrm{MPa}$. Similar to the previous results of simple supported SCW beam, the increases of the load and the yield stress resulted in the increase of the optimum weight and the decrease of the optimum weight, as shown in Figure 12.

\section{Concluding Remarks}

This study formulated an optimization problem with minimum weight as the objective function in order to propose an optimum structural design method for SCW beam, and constraint functions were composed from reviewing and comparing the design codes for shear buckling stress and the design methods proposed by the literatures of EN 19931-5 [2], DASt-R015 [4], and Pasternak and Hannebauer [1]. Global solution search method was applied to the optimum design program, and it was applied to a simple SCW model subjected to uniform load in order to investigate the convergence and efficiency of the solution by varying probabilistic parameters and genetic operators. Additionally, the effects of changing constraint function and design variable were also investigated, and the conclusions are summarized as follows.

First, an optimum structural design program of SCW beam was developed by using RCGA. The operators were selection, crossover, mutation, outbreeding, and elitist strategy, and the optimum solution based on the result of the analysis of the beam converged relatively early with greater 


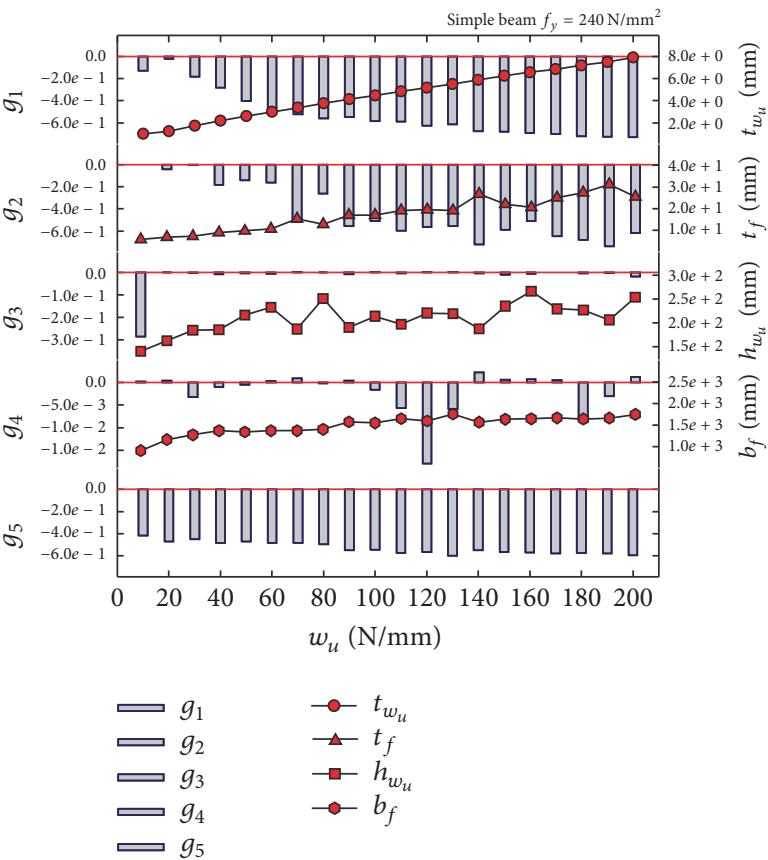

(a)



(b)

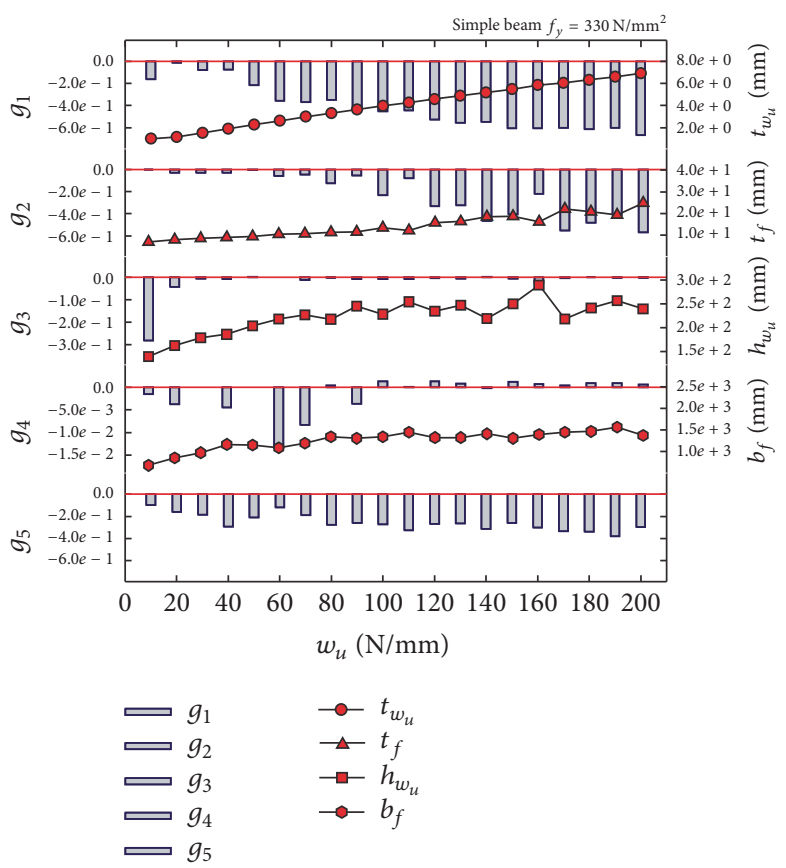

(c)

Figure 11: Variations of design variable and constraint function by external load condition. (a) $f_{y}=240 \mathrm{MPa}$. (b) $f_{y}=270 \mathrm{MPa}$. (c) $f_{y}=$ $330 \mathrm{MPa}$.

number of populations and generations. Next, the optimization method using RCGA could improve the convergence with crossover probability, mutation probability, and perturbation vectors and elite of superior trait could be attained. A stable solution could be obtained for the optimum design of the SCW beam for the crossover probability between $12.5 \%$ and $50 \%$. The best elite individual could be obtained with crossover probabilities of $25 \%$ and $37.5 \%$ when the perturbation vectors were $20 \%$ and $30 \%$, respectively. Finally, The analysis result of the optimum design program for SCW beam was deemed to reflect the proposed design method very well based on the result of varying constraint function and design variable. The increase of yield strength resulted in the reduction of optimum cross section subjected to uniform load. 


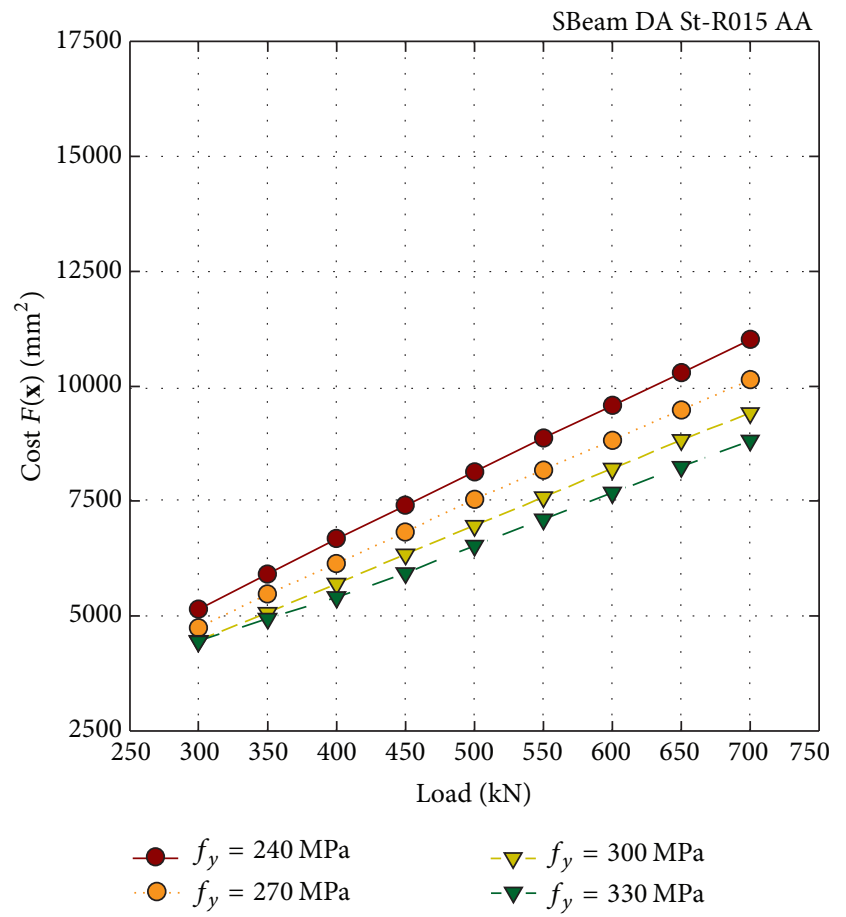

(a)



(b)

FIGURE 12: Variations of objective function by the external load type (SCW beam with fixed end). (a) Concentrated load. (b) Uniform load.

\section{Notations}

$b_{f}: \quad$ Flange plate width

$t_{f}$ : $\quad$ Flange plate thickness

$h_{w}$ : Clear web height between both flanges

$t_{w}: \quad$ The corrugated web plate thickness

$a_{3}$ : Depth of a corrugation wave

$w$ : $\quad$ Projected length of half corrugation wave

$y$ : Sinusoidal shape function of corrugation about the horizontal axis

$s$ : $\quad$ Unfolded length of corrugation for half period

$I_{x}$ : $\quad$ Moment of inertia of a half wave about the horizontal axis of symmetry

$\kappa: \quad$ Reduction factor

$\kappa_{g(\text { or } l)}:$ Reduction factor for a global (or local) web buckling

$\tau_{\mathrm{cr}, g(\text { or } l)}$ : Critical shear stress for a global (or local) web buckling

$\lambda_{c, g(\text { orl })}$ : Reference slenderness for a global (or local) web buckling

$D_{x}$ : $\quad$ Flexural stiffness of a corrugated web per unit corrugation about strong axis

$D_{y}$ : $\quad$ Flexural stiffness of a corrugated web per unit corrugation about weak axis

$V_{S d}$ : Design value of a tensile or compressive force

$V_{R d}$ : Resistance capacity of a shear force $\left(=V_{R k} / \gamma_{M}\right)$
$N_{S d}$ : Design value of a tensile or compressive force

$N_{R d}$ : Resistance capacity of a tensile or compressive force $\left(=N_{R k} / \gamma_{M}\right)$

$\gamma_{M}: \quad$ Partial safety factor

$E: \quad$ Young's modulus of elasticity

v: Poisson's ratio

$f_{y, w(\text { or } f)}$ : Yield strength of a corrugated web (or a flat flange)

$A_{\text {eff, } f}: \quad$ Effective area of the compression flange $\left(=b_{e f} \cdot t_{f}\right)$

$F(\mathbf{x})$ : Objective function of a sinusoidal corrugated web girder

$g(\mathbf{x})_{j}: \quad$ Constraint function of a sinusoidal corrugated web girder

x: Design variable vector $\left(=\left\{b_{f}, t_{f}, h_{w}, t_{w}\right\}^{T}\right)$

$A(\mathbf{x}): \quad$ Transverse cross section area of sinusoidal corrugated web beam

$\mathbf{x}^{u(\text { orl } l)}$ : Upper (or lower) boundary of a design variable vector

$\mathbf{x}^{d}: \quad$ Range of design variable $\left(=\mathbf{x}^{u}-\mathbf{x}^{l}\right)$

$\mathbf{x}^{n(\text { or } c)}: \quad$ New (or current) variable vector

$\mathbf{x}^{\text {of(or pa) }}$ : Offspring (or parent) variable vector

$d \mathbf{x}: \quad$ Perturbation vector

$\phi(\mathbf{x}): \quad$ Fitness function

$P(\mathbf{x}): \quad$ Penalty function

$r, \mathbf{r}$ : Random variable and random vector 
$p_{M}: \quad$ Probability of a mutation

$p_{C}$ : Probability of a crossover

$L: \quad$ Length of a simple beam

$\delta: \quad$ Deflection of a simple beam

$\delta_{\text {lim }}$ : Vertical deflection limit of a simple beam

$w_{u}$ : Distributed load

$M_{\max }$ : Bending moment of the distributed loaded simple beam

$V_{\text {max }}$ : Shear force of the distributed loaded simple beam.

\section{Competing Interests}

The authors declare that they have no competing interests.

\section{Acknowledgments}

This research was supported by Basic Science Research Program through the National Research Foundation of Korea (NRF) funded by the Ministry of Science, ICT \& Future Planning (NRF-2014R1A2A1A01004473).

\section{References}

[1] H. Pasternak and D. Hannebauer, "Träger mit profilierten Stegen," in Stahlbau Kalender, Ernst \& Shon, 2004.

[2] D. Annex, "Part 1-5 plated structural elements," in EUROCODE 3-Design of Steel Structures -, European Committee for Standardization (CEN), 2006.

[3] DIN 18800 Steel structures Part 1-3, German institute for standardization, Berlin, 1990.

[4] “DASt-richtlinie 015," in Träger Mit Schlanken Stegen, StahlbauVerlag, Köln, Germany, 1990.

[5] W. Siokola, Corrugated Web Beams: Technical Documentation, Zeman \& Co Gesellschaft mbH, Vienna, Austria, 1999.

[6] H. H. Abbas, Analysis and design of corrugated web i-girders for bridges using high performance steel [Ph.D. dissertation], Lehigh University, Bethlehem, Pa, USA, 2003.

[7] M. Elgaaly, A. Seshadri, and R. W. Hamilton, "Bending strength of steel beams with corrugated webs," Journal of Structural Engineering, vol. 123, no. 6, pp. 783-791, 1997.

[8] K. Anami, R. Sause, and H. H. Abbas, "Fatigue of web-flange weld of corrugated web girders: 1 . Influence of web corrugation geometry and flange geometry on web-flange weld toe stresses," International Journal of Fatigue, vol. 27, no. 4, pp. 373-381, 2005.

[9] H. H. Abbas, R. Sause, and R. G. Driver, "Simplified analysis of flange transverse bending of corrugated web I-girders under inplane moment and shear," Engineering Structures, vol. 29, no. 11, pp. 2816-2824, 2007.

[10] A. Romeijn, R. Sarkhosh, and H. de Hoop, "Basic parametric study on corrugated web girders with cut outs," Journal of Constructional Steel Research, vol. 65, no. 2, pp. 395-407, 2009.

[11] J. Moon, J.-W. Yi, B. H. Choi, and H.-E. Lee, "Lateral-torsional buckling of I-girder with corrugated webs under uniform bending," Thin-Walled Structures, vol. 47, no. 1, pp. 21-30, 2009.

[12] B. Kövesdi, B. Jáger, and L. Dunai, "Stress distribution in the flanges of girders with corrugated webs," Journal of Constructional Steel Research, vol. 79, pp. 204-215, 2012.
[13] J. T. Easley and D. E. McFarland, "Buckling of light-gage corrugated metal shear diaphragms," Journal of the Structural Division, vol. 95, no. 7, pp. 1497-1516, 1969.

[14] M. Elgaaly and H. Dagher, "Beams and girders with corrugated webs," in Proceedings of the SSRC Annual Technical Session, pp. 37-53, St. Louis, Mo, USA, April 1990.

[15] H. H. Abbas, R. Sause, and R. G. Driver, "Shear strength and stability of high performance steel corrugated web girders," in Proceedings of the Annual Stability Conference, Structural Stability Research Council, pp. 361-387, April 2002.

[16] R. G. Driver, H. H. Abbas, and R. Sause, "Shear behavior of corrugated web bridge girders," Journal of Structural Engineering, vol. 132, no. 2, pp. 195-203, 2006.

[17] J. Moon, J. Yi, B. H. Choi, and H.-E. Lee, "Shear strength and design of trapezoidally corrugated steel webs," Journal of Constructional Steel Research, vol. 65, no. 5, pp. 1198-1205, 2009.

[18] C. L. Chan, Y. A. Khalid, B. B. Sahari, and A. M. S. Hamouda, "Finite element analysis of corrugated web beams under bending," Journal of Constructional Steel Research, vol. 58, no. 11, pp. 1391-1406, 2002.

[19] W. Zhang, Y. Li, Q. Zhou, X. Qi, and G. E. O. Widera, "Optimization of the structure of an H-beam with either a flat or a corrugated web. Part 3. Development and research on H-beams with wholly corrugated webs," Journal of Materials Processing Technology, vol. 101, no. 1-3, pp. 119-123, 2000.

[20] Y. Li, W. Zhang, Q. Zhou, X. Qi, and G. E. O. Widera, "Buckling strength analysis of the web of a WCW H-beam: part 2. Development and research on H-beams with wholly corrugated webs (WCW)," Journal of Materials Processing Technology, vol. 101, no. 1, pp. 115-118, 2000.

[21] S. Shon, M. Yoo, J. Kang, and S. Lee, "Minimum weight design of sinusoidal corrugated web beam using differential evolution algorithm," International Journal of Steel Structures, vol. 15, no. 1, pp. 213-225, 2015.

[22] D. E. Goldberg, Genetic Algorithms in Search, Optimization and Machine Learning, Addison-Wesley, 1989.

[23] M. Mitchel, An Introduction to Genetic Algorithms, MIT Press, 1999.

[24] C. Fernandes, R. Tavares, and A. C. Rosa, "niGAVaPSoutbreeding in genetic algorithms," in Proceedings of the ACM Symposium on Applied Computing (SAC '00), pp. 477-482, Como, Italy, March 2000.

[25] W. Siokola and H. Poster, "Fabrication tools for corrugated web I-beams," in Modern Steel Construction, pp. 42-45, 1999. 


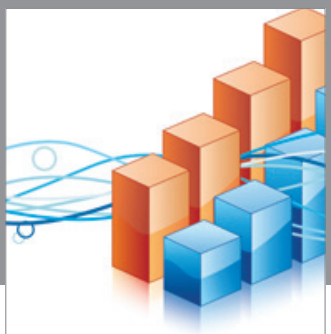

Advances in

Operations Research

vatem alat4

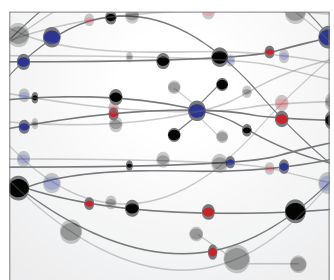

\section{The Scientific} World Journal
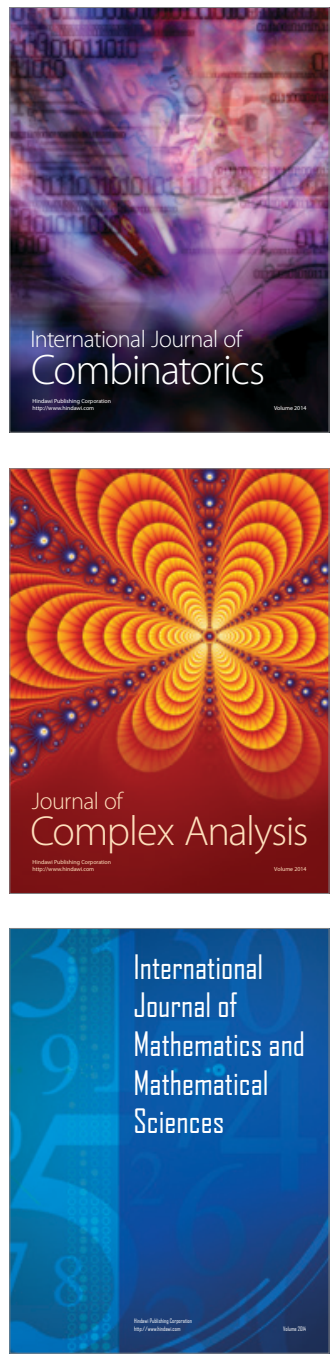
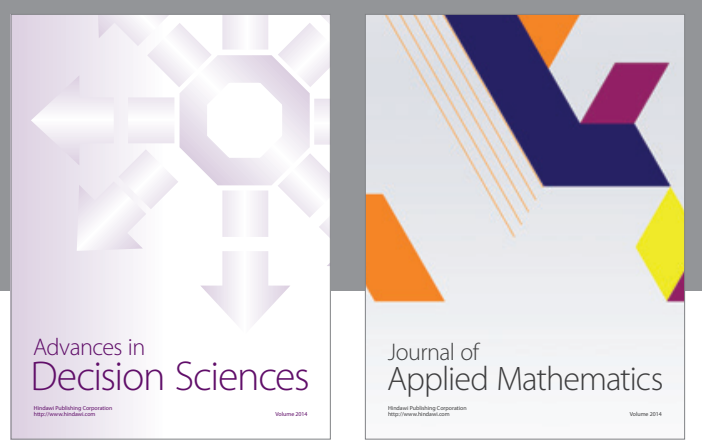

Algebra



\section{Hindawi}

Submit your manuscripts at

https://www.hindawi.com
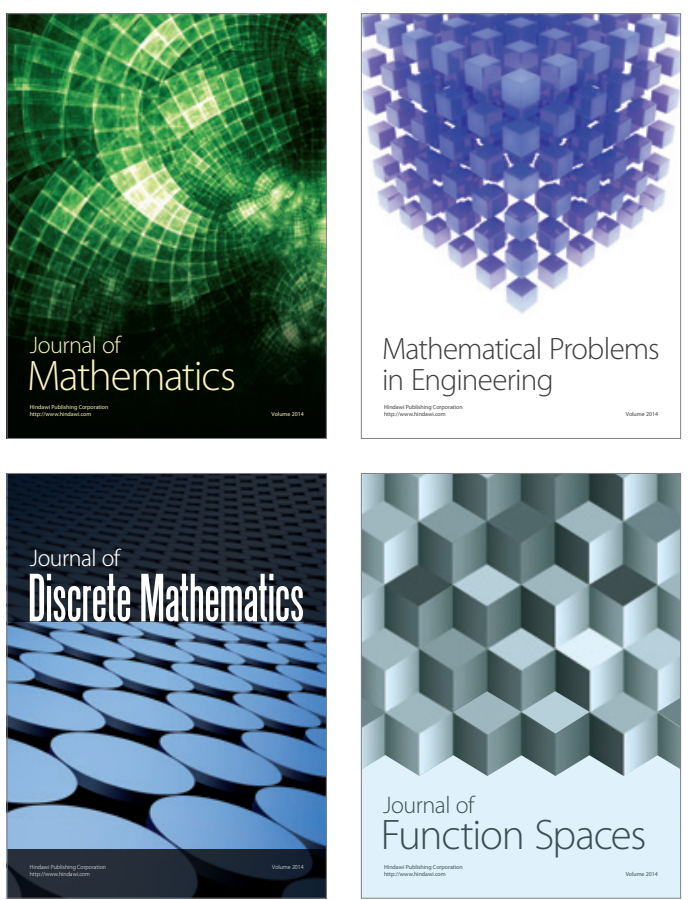

Mathematical Problems in Engineering
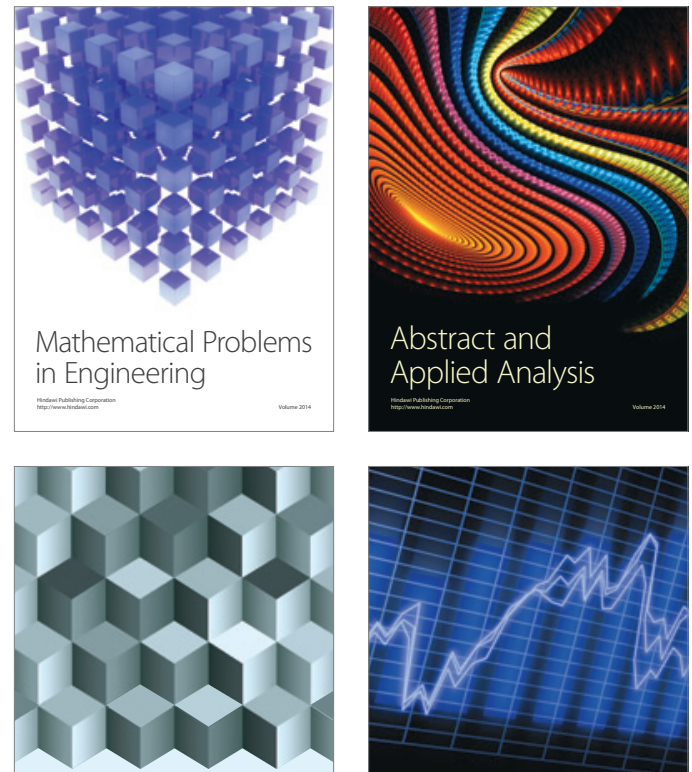

Journal of

Function Spaces

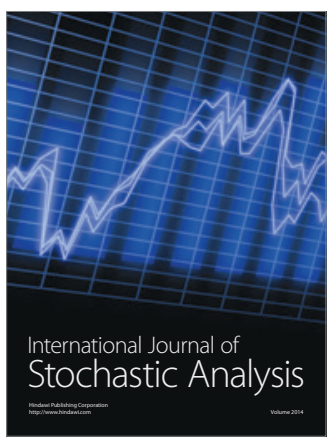

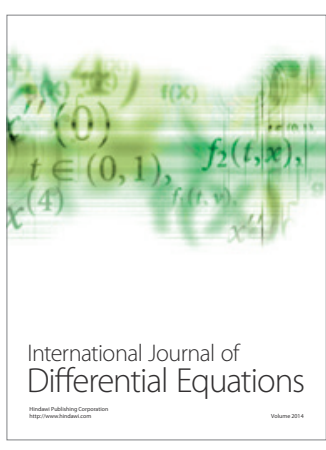
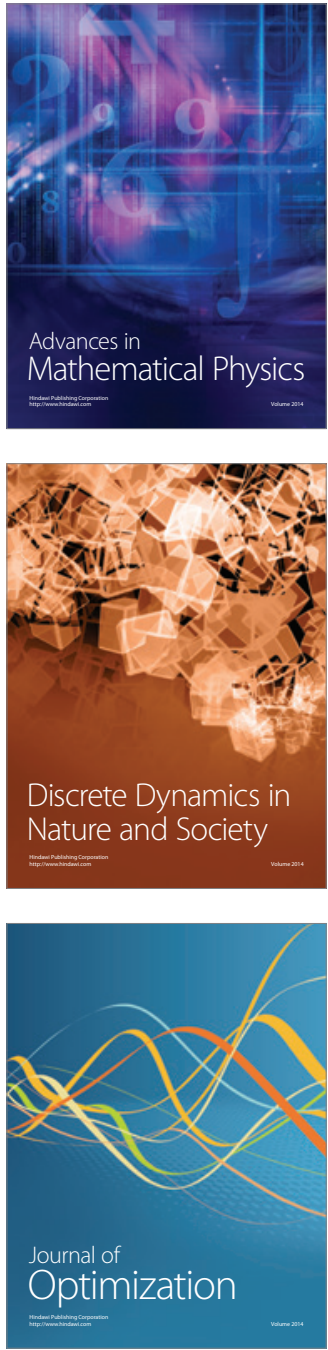